\title{
STUDY OF SOLUTIONS OF LOGARITHMIC ORDER TO HIGHER ORDER LINEAR DIFFERENTIAL-DIFFERENCE EQUATIONS WITH COEFFICIENTS HAVING THE SAME LOGARITHMIC ORDER
}

\author{
BY BENHARRAT BELAÏDI
}

\begin{abstract}
The main purpose of this paper is to study the growth of solutions of the linear differential-difference equation

$$
L(z, f)=\sum_{i=0}^{n} \sum_{j=0}^{m} A_{i j}(z) f^{(j)}\left(z+c_{i}\right)=0,
$$

where $A_{i j}(z)(i=0, \cdots, n ; j=0, \cdots, m)$ are entire or meromorphic functions of finite logarithmic order and $c_{i}(0, \cdots, n)$ are distinct complex numbers. We extend some precedent results due to $\mathrm{Wu}$ and Zheng and others.
\end{abstract}

1. Introduction and main results. Throughout this paper, we assume that readers are familiar with the standard notations and the fundamental results of the Nevanlinna value distribution theory of meromorphic functions $([\mathbf{1 2}, \mathbf{1 9}])$. Let $f$ be a meromorphic function; we define

$$
\begin{gathered}
m(r, f)=\frac{1}{2 \pi} \int_{0}^{2 \pi} \log ^{+}\left|f\left(r e^{i \varphi}\right)\right| d \varphi, \\
N(r, f)=\int_{0}^{r} \frac{n(t, f)-n(0, f)}{t} d t+n(0, f) \log r,
\end{gathered}
$$

and

$$
T(r, f)=m(r, f)+N(r, f) \quad(r>0)
$$

2010 Mathematics Subject Classification. 30D35, 34K06, 34K12.

Key words and phrases. Linear differential-difference equation, meromorphic function, logarithmic order, logarithmic type, logarithmic lower order, logarithmic lower type. 
is the Nevanlinna characteristic function of $f$, where $\log ^{+} x=\max (0, \log x)$ for $x \geq 0$, and $n(t, \infty, f)=n(t, f)$ is the number of poles of $f(z)$ lying in $|z| \leq t$, counted according to their multiplicity. Also, for $a \neq \infty$, we define

$$
\begin{gathered}
m\left(r, \frac{1}{f-a}\right)=m(r, a, f)=\frac{1}{2 \pi} \int_{0}^{2 \pi} \log ^{+} \frac{1}{\left|f\left(r e^{i \varphi}\right)-a\right|} d \varphi, \\
N\left(r, \frac{1}{f-a}\right)=N(r, a, f)=\int_{0}^{r} \frac{n(t, a, f)-n(0, a, f)}{t} d t+n(0, a, f) \log r,
\end{gathered}
$$

where $n(t, a, f)$ is the number of zeros of the equation $f(z)=a$ lying in $|z| \leq t$, counted according to their multiplicity. Also, we use the notations $\mu(f), \rho(f)$ to denote the lower order and the order of a meromorphic function $f$.

To express the rate of growth of meromorphic solutions of infinite order, we recall the following definition.

Definition $1.1([\mathbf{1 6}, \mathbf{1 9}])$. Let $f$ be a meromorphic function. Then the hyper-order $\rho_{2}(f)$ of $f(z)$ is defined by

$$
\rho_{2}(f)=\limsup _{r \rightarrow+\infty} \frac{\log \log T(r, f)}{\log r} .
$$

If $f$ is an entire function, then the hyper-order of $f(z)$ is defined as

$$
\rho_{2}(f)=\limsup _{r \rightarrow+\infty} \frac{\log \log T(r, f)}{\log r}=\limsup _{r \rightarrow+\infty} \frac{\log \log \log M(r, f)}{\log r},
$$

where $M(r, f)$ is the maximum modulus of $f$ in the circle $|z|=r$.

Definition $1.2([\mathbf{1 2}])$. Let $f$ be an entire function of order $\rho(0<\rho<$ $+\infty)$. The type of $f$ is defined as

$$
\tau(f)=\limsup _{r \rightarrow+\infty} \frac{\log M(r, f)}{r^{\rho}} .
$$

Similarly, the lower type of an entire function $f$ of lower order $\mu(0<\mu<\infty)$ is defined by

$$
\underline{\tau}(f)=\liminf _{r \rightarrow+\infty} \frac{\log M(r, f)}{r^{\mu}} .
$$

Definition $1.3([\mathbf{1 2},[\mathbf{1 9}])$. For $a \in \overline{\mathbb{C}}=\mathbb{C} \cup\{\infty\}$, the deficiency of $a$ with respect to a meromorphic function $f$ is defined as

$$
\begin{gathered}
\delta(a, f)=\liminf _{r \rightarrow+\infty} \frac{m\left(r, \frac{1}{f-a}\right)}{T(r, f)}=1-\limsup _{r \rightarrow+\infty} \frac{N\left(r, \frac{1}{f-a}\right)}{T(r, f)}, a \neq \infty, \\
\delta(\infty, f)=\liminf _{r \rightarrow+\infty} \frac{m(r, f)}{T(r, f)}=1-\limsup _{r \rightarrow+\infty} \frac{N(r, f)}{T(r, f)} .
\end{gathered}
$$


Recently, the difference counterparts of Nevanlinna theory have been established. The key result is the difference analogue of the lemma on the logarithmic derivative obtained by Halburd-Korhonen [10] and Chiang-Feng [6], independently. Subsequently Halburd and Korhonen [11] showed how all key results of the Nevanlinna theory have corresponding difference variants as well. After that, it was with a growing interest that solutions to difference equations in the complex domain have been investigated by making use of this variant of the value distribution theory, see $[\mathbf{4}, \mathbf{1 5}, \mathbf{1 7}, \mathbf{1 8}, \mathbf{2 0}]$. In [15], Laine and Yang considered complex linear difference equations and obtained the following theorem.

Theorem A $\left([\mathbf{1 5} \mid)\right.$. Let $A_{0}(z), A_{1}(z), \cdots, A_{n}(z)$ be entire functions of $f-$ nite order such that among those having the maximal order $\rho=\max _{0 \leq j \leq n}\left\{\rho\left(A_{j}\right)\right\}$, there is exactly one whose type is strictly greater than the others'. Then for any meromorphic solution of

$A_{n}(z) f(z+n)+A_{n-1}(z) f(z+n-1)+\cdots+A_{1}(z) f(z+1)+A_{0}(z) f(z)=0$, we have $\rho(f) \geq \rho+1$.

In [16], Tu and Yi investigated the growth of solutions of a class of higher order linear differential equations with entire coefficients when most of them are of the same order, and obtained the following result.

Theorem B $([\mathbf{1 6}])$. Let $A_{0}(z), \cdots, A_{k-1}(z)$ be entire functions such that $\rho\left(A_{0}\right)=\rho(0<\rho<+\infty)$ and $\tau\left(A_{0}\right)=\tau(0<\tau<+\infty)$, and let $\rho\left(A_{j}\right) \leq$ $\rho\left(A_{0}\right)=\rho(j=1,2, \cdots, k-1), \tau\left(A_{j}\right)<\tau\left(A_{0}\right)=\tau(j=1,2, \cdots, k-1)$ if $\rho\left(A_{j}\right)=\rho\left(A_{0}\right)(j=1,2, \cdots, k-1)$. Then every solution $f \not \equiv 0$ of

$$
f^{(k)}+A_{k-1}(z) f^{(k-1)}+\cdots+A_{1}(z) f^{\prime}+A_{0}(z) f=0,
$$

satisfies $\rho(f)=+\infty$ and $\rho_{2}(f)=\rho\left(A_{0}\right)=\rho$.

From Theorems $\mathrm{A}$ and $\mathrm{B}$, we deduce that when there is exactly one dominant coefficient among those coefficients having the same maximal order, we may obtain the growth relation between the solutions and the coefficients of the above complex linear difference equation or complex linear differential equation. In recent paper [18, Wu and Zheng investigated the growth of meromorphic solutions of the linear differential-difference equation

$$
L(z, f)=\sum_{i=0}^{n} \sum_{j=0}^{m} A_{i j}(z) f^{(j)}\left(z+c_{i}\right)=0,
$$

where $A_{i j}(z)(i=0, \cdots, n ; j=0, \cdots, m)$ are entire or meromorphic functions of finite order and $c_{i}(0, \cdots, n)$ are distinct complex numbers, where there is only one dominant coefficient. Hence, from Theorems $\mathrm{A}$ and $\mathrm{B}$ a natural 
question emerges: How to express the growth of solutions of (1.1) when all coefficients $A_{0}(z), A_{1}(z), \cdots, A_{n}(z)$ are entire or meromorphic functions and of order zero in $\mathbb{C}$ ? The main purpose of this paper is to make use the concept of finite logarithmic order due to Chern [5] to extend previous results of Wu and Zheng $\mathbf{1 8}$ for meromorphic solutions to equation 1.1) of zero order in $\mathbb{C}$. We recall the following definitions.

Definition $1.4(\sqrt[5]{\mathbf{5}})$. The logarithmic order of a meromorphic function $f$ is defined as

$$
\rho_{\log }(f)=\limsup _{r \rightarrow+\infty} \frac{\log T(r, f)}{\log \log r} .
$$

If $f$ is an entire function, then

$$
\rho_{\log }(f)=\limsup _{r \rightarrow+\infty} \frac{\log \log M(r, f)}{\log \log r} .
$$

REMARK 1.1. Obviously, the logarithmic order of any non-constant rational function $f$ is one, and thus, any transcendental meromorphic function in the plane has logarithmic order no less than one. However, a function of logarithmic order one is not necessarily a rational function. Constant functions have zero logarithmic order, while there are no meromorphic functions of logarithmic order between zero and one. Moreover, any meromorphic function with finite logarithmic order in the plane is of order zero.

Definition 1.5. The logarithmic lower order of a meromorphic function $f$ is defined as

$$
\mu_{\log }(f)=\liminf _{r \rightarrow+\infty} \frac{\log T(r, f)}{\log \log r} .
$$

If $f$ is an entire function, then

$$
\mu_{\log }(f)=\liminf _{r \rightarrow+\infty} \frac{\log \log M(r, f)}{\log \log r} .
$$

DEFInition $1.6([\mathbf{3}])$. The logarithmic type of an entire function $f$ with $1 \leq \rho_{\log }(f)<+\infty$ is defined by

$$
\tau_{\log }(f)=\limsup _{r \rightarrow+\infty} \frac{\log M(r, f)}{(\log r)^{\rho_{\log }(f)}} .
$$

Similarly the logarithmic lower type of an entire function $f$ with $1 \leq \mu_{\log }(f)<$ $+\infty$ is defined by

$$
\underline{\tau}_{\log }(f)=\liminf _{r \rightarrow+\infty} \frac{\log M(r, f)}{(\log r)^{\mu_{\log }(f)}} .
$$


REMARK 1.2. It is evident that the logarithmic type of any non-constant polynomial $P$ equals its degree $\operatorname{deg}(P)$; that any non-constant rational function is of finite logarithmic type, and that any transcendental meromorphic function whose logarithmic order equals one in the plane must be of infinite logarithmic type.

Recently, the concept of logarithmic order has been used to investigate the growth and the oscillation of solutions of linear differential equations in the complex plane $[3$ and complex linear difference and $q$-difference equations in the complex plane and in the unit disc $([\mathbf{1}, \mathbf{2}, \mathbf{1 3}, \mathbf{1 4}, \mathbf{1 7}])$. In what follows, we consider the growth estimates of meromorphic solutions of the homogeneous equation (1.1) with some coefficients having the same maximal order or maximal lower order, and we obtain the following results.

Theorem 1.1. Let $A_{i j}(z)(i=0, \cdots, n ; j=0, \cdots, m)$ be entire functions such that there exists an integer $s(0 \leq s \leq n)$ satisfying

$$
\max \left\{\rho_{\log }\left(A_{i j}\right):(i, j) \neq(s, 0)\right\} \leq \rho_{\log }\left(A_{s 0}\right)<\infty,
$$

and

$$
\max \left\{\tau_{\log }\left(A_{i j}\right): \rho_{\log }\left(A_{i j}\right)=\rho_{\log }\left(A_{s 0}\right), \quad(i, j) \neq(s, 0)\right\}<\tau_{\log }\left(A_{s 0}\right) .
$$

Then every meromorphic solution $f \not \equiv 0$ of equation (1.1) satisfies $\rho_{\log }(f) \geq$ $\rho_{\log }\left(A_{s 0}\right)+1$.

TheOREM 1.2. Let $A_{i j}(z)(i=0, \cdots, n ; j=0, \cdots, m)$ be entire functions such that there exists an integer $s(0 \leq s \leq n)$ satisfying

$$
\max \left\{\rho_{\log }\left(A_{i j}\right):(i, j) \neq(s, 0)\right\} \leq \mu_{\log }\left(A_{s 0}\right)<\infty,
$$

and

$$
\max \left\{\tau_{\log }\left(A_{i j}\right): \rho_{\log }\left(A_{i j}\right)=\mu_{\log }\left(A_{s 0}\right), \quad(i, j) \neq(s, 0)\right\}<\underline{\tau}_{\log }\left(A_{s 0}\right) .
$$

Then every meromorphic solution $f \not \equiv 0$ of equation (1.1) satisfies $\mu_{\log }(f) \geq$ $\mu_{\log }\left(A_{s 0}\right)+1$.

THEOREM 1.3. Let $H$ be a set of complex numbers satisfying $\overline{\log d e n s}\{|z|$ : $z \in H\}>0$, and let $A_{i j}(z)(i=0, \cdots, n ; j=0, \cdots, m)$ be entire functions satisfying $\max \left\{\rho_{\log }\left(A_{i j}\right):(i=0, \cdots, n ; j=0, \cdots, m)\right\} \leq \rho$ with $1 \leq \rho<$ $+\infty$. If there exists an integer $s(0 \leq s \leq n)$ such that for some constants $0 \leq \beta<\alpha$ and sufficiently small $\varepsilon(0<\varepsilon<\rho)$, we have

$$
\left|A_{s 0}(z)\right| \geq \exp \left\{\alpha[\log r]^{\rho-\varepsilon}\right\}
$$

and

$$
\left|A_{i j}(z)\right| \leq \exp \left\{\beta[\log r]^{\rho-\varepsilon}\right\}, \quad(i, j) \neq(s, 0)
$$

as $|z|=r \rightarrow+\infty$ for $z \in H$, then every meromorphic solution $f \not \equiv 0$ of equation (1.1) satisfies $\rho_{\log }(f) \geq \rho_{\log }\left(A_{s 0}\right)+1$. 
REMARK 1.3. By the assumptions of Theorem 1.3, we obtain $\rho_{\log }\left(A_{s 0}\right)=\rho$. Indeed, we have $\rho_{\log }\left(A_{s 0}\right) \leq \rho$. Suppose that $\rho_{\log }\left(A_{s 0}\right)=\mu<\rho$. Then, by Definition 1.4 and (1.6), we have for any given $\varepsilon\left(0<\varepsilon<\frac{\rho-\mu}{2}\right)$

$$
\exp \left\{\alpha[\log r]^{\rho-\varepsilon}\right\} \leq\left|A_{s 0}(z)\right| \leq \exp \left\{[\log r]^{\mu+\varepsilon}\right\} .
$$

as $|z|=r \rightarrow+\infty$ for $z \in H$. By $\varepsilon\left(0<\varepsilon<\frac{\rho-\mu}{2}\right)$ this is a contradiction as $r \rightarrow+\infty$. Hence $\rho_{\log }\left(A_{s 0}\right)=\rho$.

Theorem 1.4. Let $A_{i j}(z)(i=0, \cdots, n ; j=0, \cdots, m)$ be entire functions of finite logarithmic order such that there exists an integer $s(0 \leq s \leq n)$ satisfying

$$
\limsup _{r \rightarrow+\infty} \frac{\sum_{(i, j) \neq(s, 0)} m\left(r, A_{i j}\right)}{m\left(r, A_{s 0}\right)}<1 .
$$

Then every meromorphic solution $f \not \equiv 0$ of equation (1.1) satisfies $\rho_{\log }(f) \geq$ $\rho_{\log }\left(A_{s 0}\right)+1$.

The following theorems give some properties of the logarithmic order of meromorphic solutions of (1.1) in the case when the coefficients are meromorphic functions.

TheOREM 1.5. Let $A_{i j}(z)(i=0, \cdots, n ; j=0, \cdots, m)$ be meromorphic functions such that there exists an integer $s(0 \leq s \leq n)$ satisfying $\rho_{\log }\left(A_{s 0}\right)>$ $\max \left\{\rho_{\log }\left(A_{i j}\right):(i, j) \neq(s, 0)\right\}$ and $\delta\left(\infty, A_{s 0}\right)>0$. Then every meromorphic solution $f \not \equiv 0$ of equation (1.1) satisfies $\rho_{\log }(f) \geq \rho_{\log }\left(A_{s 0}\right)+1$.

Theorem 1.6. Let $A_{i j}(z)(i=0, \cdots, n ; j=0, \cdots, m)$ be meromorphic functions of finite logarithmic order such that there exists an integer $s$ $(0 \leq s \leq n)$ satisfying $\limsup _{r \rightarrow+\infty} \frac{\sum_{(i, j) \neq(s, 0)} m\left(r, A_{i j}\right)}{m\left(r, A_{s 0}\right)}<1$ and $\delta\left(\infty, A_{s 0}\right)>0$.

Then every meromorphic solution $f \not \equiv 0$ of equation (1.1) satisfies $\rho_{\log }(f) \geq$ $\rho_{\log }\left(A_{s 0}\right)+1$.

2. Some lemmas. We recall the following definitions. The linear measure of a set $E \subset(0,+\infty)$ is defined as $m(E)=\int_{0}^{+\infty} \chi_{E}(t) d t$ and the logarithmic measure of a set $F \subset(1,+\infty)$ is defined by $\operatorname{lm}(F)=\int_{1}^{+\infty} \frac{\chi_{F}(t)}{t} d t$, where $\chi_{H}(t)$ is the characteristic function of a set $H$. The upper density of a set $E \subset(0,+\infty)$ is defined by

$$
\overline{\operatorname{dens}} E=\limsup _{r \longrightarrow+\infty} \frac{m(E \cap[0, r])}{r} .
$$

The upper logarithmic density of a set $F \subset(1,+\infty)$ is defined by

$$
\overline{\log d e n s}(F)=\limsup _{r \longrightarrow+\infty} \frac{\operatorname{lm}(F \cap[1, r])}{\log r} .
$$


It is easy to obtain the following remark.

REMARK 2.1. For all $H \subset[1,+\infty)$ the following statements hold:

i) If $\operatorname{lm}(H)=\infty$, then $m(H)=\infty$;

ii) If $\overline{\operatorname{dens}} H>0$, then $m(H)=\infty$;

iii) If $\overline{\log d e n s} H>0$, then $\operatorname{lm}(H)=\infty$.

LEMMA $2.1(\sqrt{8})$. Let $f(z)$ be a transcendental meromorphic function in the plane, and let $\alpha>1$ be a given constant. Then there exist a set $E_{1} \subset(1,+\infty)$ of finite logarithmic measure, and a constant $B>0$ depending only on $\alpha$ and $(m, n)(m, n \in\{0,1, \cdots, k\}) m<n$ such that for all $z$ with $|z|=r \notin[0,1] \cup E_{1}$, we have

$$
\left|\frac{f^{(n)}(z)}{f^{(m)}(z)}\right| \leq B\left(\frac{T(\alpha r, f)}{r}\left(\log ^{\alpha} r\right) \log T(\alpha r, f)\right)^{n-m} .
$$

From the above lemma, we obtain the following result.

LEMMA 2.2. Let $f(z)$ be a transcendental meromorphic function in the plane with $1 \leq \rho_{\log }(f)=\rho<+\infty$, and let $\varepsilon>0, \alpha>1$ be given constants. Then there exist a set $E_{2} \subset(1,+\infty)$ of finite logarithmic measure, and $(m, n)$ $(m, n \in\{0,1, \cdots, k\}) m<n$ such that for all $z$ with $|z|=r \notin[0,1] \cup E_{2}$, we have

$$
\left|\frac{f^{(n)}(z)}{f^{(m)}(z)}\right| \leq\left(\frac{(\log r)^{\rho+\alpha+\varepsilon}}{r}\right)^{n-m} .
$$

Proof. Since $f(z)$ has finite logarithmic order $\rho_{\log }(f)=\rho<+\infty$, so given $\varepsilon(0<\varepsilon<2)$ and sufficiently large $r>R$, we have

$$
T(r, f)<(\log r)^{\rho+\frac{\varepsilon}{2}} .
$$

Combining (2.1) with Lemma 2.1, for $\alpha>1$, there exist a set $E_{2}=[0, R] \cup E_{1}$ of finite logarithmic measure and a constant $B>0$, such that if $|z|=r \notin$ $[0,1] \cup E_{2}$, we obtain

$$
\begin{aligned}
\left|\frac{f^{(n)}(z)}{f^{(m)}(z)}\right| & \leq B\left(\frac{(\log \alpha r)^{\rho+\frac{\varepsilon}{2}}}{r}\left(\log ^{\alpha} r\right) \log (\log \alpha r)^{\rho+\frac{\varepsilon}{2}}\right)^{n-m} \\
& \leq\left(\frac{(\log r)^{\rho+\alpha+\varepsilon}}{r}\right)^{n-m} .
\end{aligned}
$$

REMARK 2.2. It is shown in 7 , p. 66], that for an arbitrary complex number $c \neq 0$, the following inequalities

$$
(1+o(1)) T(r-|c|, f(z)) \leq T(r, f(z+c)) \leq(1+o(1)) T(r+|c|, f(z))
$$


hold as $r \rightarrow+\infty$ for an arbitrary meromorphic function $f(z)$. Therefore, it is easy to obtain

$$
\rho_{\log }(f+c)=\rho_{\log }(f), \quad \mu_{\log }(f+c)=\mu_{\log }(f) .
$$

Lemma $2.3\left([\mathbf{1})\right.$. Let $\eta_{1}, \eta_{2}$ be two arbitrary complex numbers such that $\eta_{1} \neq \eta_{2}$ and let $f(z)$ be a finite logarithmic order meromorphic function. Let $\rho$ be the logarithmic order of $f(z)$. Then for each $\varepsilon>0$, we have

$$
m\left(r, \frac{f\left(z+\eta_{1}\right)}{f\left(z+\eta_{2}\right)}\right)=O\left((\log r)^{\rho-1+\varepsilon}\right) .
$$

LEMma $2.4(\sqrt{\mathbf{6}} \mid)$. Let $f$ be a meromorphic function, $\eta$ a non-zero complex number, and let $\gamma>1$, and $\varepsilon>0$ be given real constants. Then there exist a subset $E_{3} \subset(1,+\infty)$ of finite logarithmic measure, and a constant $A$ depending only on $\gamma$ and $\eta$, such that for all $|z|=r \notin E_{3} \cup[0,1]$, we have

$$
|\log | \frac{f(z+\eta)}{f(z)}|| \leq A\left(\frac{T(\gamma r, f)}{r}+\frac{n(\gamma r)}{r} \log ^{\gamma} r \log ^{+} n(\gamma r)\right),
$$

where $n(t)=n(t, \infty, f)+n(t, \infty, 1 / f)$.

Lemma $2.5([\mathbf{8})$. Let $f$ be a transcendental meromorphic function, let $j$ be a non-negative integer, let a be a value in the extended complex plane, and let $\alpha>1$ be a real constant. Then there exists a constant $R>0$ such that for all $r>R$, we have

$$
n\left(r, a, f^{(j)}\right) \leq \frac{2 j+6}{\log \alpha} T(\alpha r, f) .
$$

LEMMA 2.6. Let $f$ be a meromorphic function with $1 \leq \mu_{\log }(f)<+\infty$. Then there exists a set $E_{4} \subset(1,+\infty)$ of infinite logarithmic measure such that for $r \in E_{4} \subset(1,+\infty)$, we have

$$
T(r, f)<(\log r)^{\mu_{\log }(f)+\varepsilon} .
$$

Proof. By definition of the logarithmic lower order, there exists a sequence $\left\{r_{n}\right\}_{n=1}^{\infty}$ tending to $\infty$, satisfying $\left(1+\frac{1}{n}\right) r_{n}<r_{n+1}$ and

$$
\lim _{r_{n} \rightarrow \infty} \frac{\log T\left(r_{n}, f\right)}{\log \log r_{n}}=\mu_{\log }(f) .
$$

Then for any given $\varepsilon>0$, there exists an integer $n_{1}$ such that for all $n \geq n_{1}$,

$$
T\left(r_{n}, f\right)<\left(\log r_{n}\right)^{\mu_{\log }(f)+\frac{\varepsilon}{2}} .
$$

Set $E_{4}=\bigcup_{n=n_{1}}^{\infty}\left[\frac{n}{n+1} r_{n}, r_{n}\right]$. Then for $r \in E_{4} \subset(1,+\infty)$, we obtain $T(r, f) \leq T\left(r_{n}, f\right)<\left(\log r_{n}\right)^{\mu_{\log }(f)+\frac{\varepsilon}{2}} \leq\left(\log \frac{n+1}{n} r\right)^{\mu_{\log }(f)+\frac{\varepsilon}{2}}<(\log r)^{\mu_{\log }(f)+\varepsilon}$, 
and $\operatorname{lm}\left(E_{4}\right)=\sum_{n=n_{1}}^{\infty} \int_{n+1}^{r_{n}} \frac{d t}{t}=\sum_{n=n_{1}}^{\infty} \log \left(1+\frac{1}{n}\right)=\infty$. Thus, Lemma 2.6 is proved.

Lemma 2.7. Let $f$ be a meromorphic function, $\eta$ a non-zero complex number, and $\varepsilon>0, \beta>1$ be given real constants. Then there exists a subset $E_{5} \subset(1,+\infty)$ of finite logarithmic measure, such that if $f$ has finite logarithmic order $\rho$, then for all $|z|=r \notin E_{5} \cup[0,1]$, we have

$$
\exp \left\{-\frac{(\log r)^{\rho+\beta+\varepsilon}}{r}\right\} \leq\left|\frac{f(z+\eta)}{f(z)}\right| \leq \exp \left\{\frac{(\log r)^{\rho+\beta+\varepsilon}}{r}\right\} .
$$

Proof. By Lemma 2.4 there exist a subset $E_{5} \subset(1,+\infty)$ of finite logarithmic measure, and a constant $A$ depending only on $\gamma$ and $\eta$, such that for all $|z|=r \notin E_{5} \cup[0,1]$, we have

$$
|\log | \frac{f(z+\eta)}{f(z)}|| \leq A\left(\frac{T(\gamma r, f)}{r}+\frac{n(\gamma r)}{r} \log ^{\gamma} r \log ^{+} n(\gamma r)\right),
$$

where $n(t)=n(t, \infty, f)+n(t, \infty, 1 / f)$. By using 2.3 and 2.5), we obtain

$$
\begin{aligned}
|\log | \frac{f(z+\eta)}{f(z)}|| \leq & A\left(\frac{T(\gamma r, f)}{r}\right. \\
& \left.+\frac{12}{\log \alpha} \frac{T(\alpha \gamma r, f)}{r} \log ^{\gamma} r \log ^{+}\left(\frac{12}{\log \alpha} T(\alpha \gamma r, f)\right)\right) \\
\leq & B\left(T(\beta r, f) \frac{\log ^{\beta} r}{r} \log T(\beta r, f)\right),
\end{aligned}
$$

where $B>0$ is some constant and $\beta=\alpha \gamma>1$. Since $f(z)$ has finite logarithmic order $\rho_{\log }(f)=\rho<+\infty$, so given $\varepsilon, 0<\varepsilon<2$, for sufficiently large $r$, we have

$$
T(r, f)<(\log r)^{\rho+\frac{\varepsilon}{2}} .
$$

Then by using (2.6) and (2.7), we obtain

$$
\begin{aligned}
|\log | \frac{f(z+\eta)}{f(z)}|| & \leq B\left(T(\beta r, f) \frac{\log ^{\beta} r}{r} \log T(\beta r, f)\right) \\
& \leq B(\log \beta r)^{\rho+\frac{\varepsilon}{2}} \frac{\log ^{\beta} r}{r} \log (\log \beta r)^{\rho+\frac{\varepsilon}{2}} \leq \frac{(\log r)^{\rho+\beta+\varepsilon}}{r} .
\end{aligned}
$$

From 2.8), we easily obtain 2.4.

Lemma 2.8. Let $\eta_{1}, \eta_{2}$ be two arbitrary complex numbers such that $\eta_{1} \neq \eta_{2}$ and let $f(z)$ be a meromorphic function of finite logarithmic order $\rho$. Let 
$\varepsilon>0$ and $\beta>1$ be given. Then there exists a subset $E_{6} \subset(1,+\infty)$ of finite logarithmic measure such that for all $|z|=r \notin E_{6}$, we have

$$
\exp \left\{-\frac{(\log r)^{\rho+\beta+\varepsilon}}{r}\right\} \leq\left|\frac{f\left(z+\eta_{1}\right)}{f\left(z+\eta_{2}\right)}\right| \leq \exp \left\{\frac{(\log r)^{\rho+\beta+\varepsilon}}{r}\right\} .
$$

Proof. We can write

$$
\left|\frac{f\left(z+\eta_{1}\right)}{f\left(z+\eta_{2}\right)}\right|=\left|\frac{f\left(z+\eta_{2}+\eta_{1}-\eta_{2}\right)}{f\left(z+\eta_{2}\right)}\right| \quad\left(\eta_{1} \neq \eta_{2}\right) .
$$

Then by using Lemma 2.7, for any given $\varepsilon>0, \beta>1$ and all $\left|z+\eta_{2}\right|=R \notin$ $E_{5} \cup[0,1]$, such that $\operatorname{lm}\left(E_{5}\right)<\infty$, we obtain

$$
\begin{aligned}
\exp \left\{-\frac{(\log r)^{\rho+\beta+\varepsilon}}{r}\right\} & \leq \exp \left\{-\frac{\left(\log \left(|z|+\left|\eta_{2}\right|\right)\right)^{\rho+\beta+\frac{\varepsilon}{2}}}{\left|z+\eta_{2}\right|}\right\} \\
& =\exp \left\{-\frac{(\log R)^{\rho+\beta+\frac{\varepsilon}{2}}}{R}\right\} \leq\left|\frac{f\left(z+\eta_{1}\right)}{f\left(z+\eta_{2}\right)}\right| \\
& =\left|\frac{f\left(z+\eta_{2}+\eta_{1}-\eta_{2}\right)}{f\left(z+\eta_{2}\right)}\right| \leq \exp \left\{\frac{(\log R)^{\rho+\beta+\frac{\varepsilon}{2}}}{R}\right\} \\
& \leq \exp \left\{\frac{\left(\log \left(|z|+\left|\eta_{2}\right|\right)\right)^{\rho+\beta+\frac{\varepsilon}{2}}}{\left|z+\eta_{2}\right|}\right\} \leq \exp \left\{\frac{(\log r)^{\rho+\beta+\varepsilon}}{r}\right\},
\end{aligned}
$$

where $|z|=r \notin E_{6}$ and $E_{6}$ is a set of finite logarithmic measure.

By using Lemmas 2.4 2.6, we can generalize Lemma 2.2 and Lemma 2.8 into finite logarithmic lower order case as following.

LEMMA 2.9. Let $f(z)$ be a transcendental meromorphic function in the plane with $1 \leq \mu_{\log }(f)=\mu<+\infty$, and let $\varepsilon>0, \alpha>1$ be given constants. Then there exist a set $E_{7} \subset(1,+\infty)$ of infinite logarithmic measure, and $(m, n)$ $(m, n \in\{0,1, \cdots, k\}) m<n$ such that for all $z$ with $|z|=r \in E_{7}$, we have

$$
\left|\frac{f^{(n)}(z)}{f^{(m)}(z)}\right| \leq\left(\frac{(\log r)^{\mu+\alpha+\varepsilon}}{r}\right)^{n-m} .
$$

LEMMA 2.10. Let $\eta_{1}, \eta_{2}$ be two arbitrary complex numbers such that $\eta_{1} \neq \eta_{2}$ and let $f(z)$ be a meromorphic function of finite logarithmic lower order $\mu$. Let $\varepsilon>0$ and $\beta>1$ be given. Then there exists a subset $E_{8} \subset(1,+\infty)$ of infinite logarithmic measure such that for all $|z|=r \in E_{8}$, we have

$$
\exp \left\{-\frac{(\log r)^{\mu+\beta+\varepsilon}}{r}\right\} \leq\left|\frac{f\left(z+\eta_{1}\right)}{f\left(z+\eta_{2}\right)}\right| \leq \exp \left\{\frac{(\log r)^{\mu+\beta+\varepsilon}}{r}\right\} .
$$


Lemma $2.11(\sqrt{\mathbf{1}})$. Let $f$ be a meromorphic function with $\rho_{\log }(f) \geq 1$. Then there exists a set $E_{9} \subset(1,+\infty)$ of infinite logarithmic measure such that

$$
\lim _{\substack{r \rightarrow+\infty \\ r \in E_{9}}} \frac{\log T(r, f)}{\log \log r}=\rho .
$$

Lemma $2.12\left([\sqrt[\mathbf{1}]{]})\right.$. Let $f_{1}, f_{2}$ be meromorphic functions satisfying $\rho_{\log }\left(f_{1}\right)>$ $\rho_{\log }\left(f_{2}\right)$. Then there exists a set $E_{10} \subset(1,+\infty)$ of infinite logarithmic measure such that for all $r \in E_{10}$, we have

$$
\lim _{r \rightarrow+\infty} \frac{T\left(r, f_{2}\right)}{T\left(r, f_{1}\right)}=0
$$

Lemma 2.13. Let $f$ be an entire function with $1 \leq \mu_{\log }(f)<+\infty$. Then there exists a set $E_{11} \subset(1,+\infty)$ of infinite logarithmic measure such that

$$
\underline{\tau}_{\log }(f)=\lim _{\substack{r \rightarrow+\infty \\ r \in E_{11}}} \frac{\log M(r, f)}{(\log r)^{\mu_{\log }(f)}} .
$$

Proof. By the definition of the logarithmic lower type, there exists a sequence $\left\{r_{n}\right\}_{n=1}^{\infty}$ tending to $\infty$, satisfying $\left(1+\frac{1}{n}\right) r_{n}<r_{n+1}$, and

$$
\underline{\tau}_{\log }(f)=\lim _{r_{n} \rightarrow \infty} \frac{\log M\left(r_{n}, f\right)}{\left(\log r_{n}\right)^{\mu_{\log }(f)}} .
$$

Then for any given $\varepsilon>0$, there exists an $n_{1}$ such that for $n \geq n_{1}$ and any $r \in\left[\frac{n}{n+1} r_{n}, r_{n}\right]$, we have

$$
\frac{\log M\left(\frac{n}{n+1} r_{n}, f\right)}{\left(\log r_{n}\right)^{\mu_{\log }(f)}} \leq \frac{\log M(r, f)}{(\log r)^{\mu_{\log }(f)}} \leq \frac{\log M\left(r_{n}, f\right)}{\left(\log \frac{n}{n+1} r_{n}\right)^{\mu_{\log }(f)}} .
$$

It follows that

$$
\begin{gathered}
\left(\frac{\log \frac{n}{n+1} r_{n}}{\log r_{n}}\right)^{\mu_{\log }(f)} \frac{\log M\left(\frac{n}{n+1} r_{n}, f\right)}{\left(\log \frac{n}{n+1} r_{n}\right)^{\mu_{\log }(f)}} \leq \frac{\log M(r, f)}{(\log r)^{\mu_{\log }(f)}} \\
\leq \frac{\log M\left(r_{n}, f\right)}{\left(\log r_{n}\right)^{\mu_{\log }(f)}}\left(\frac{\log r_{n}}{\log \frac{n}{n+1} r_{n}}\right)^{\mu_{\log }(f)}
\end{gathered}
$$

Set

$$
E_{11}=\bigcup_{n=n_{1}}^{\infty}\left[\frac{n}{n+1} r_{n}, r_{n}\right]
$$

Then we have

$$
\lim _{\substack{r \rightarrow+\infty \\ r \in E_{11}}} \frac{\log M(r, f)}{(\log r)^{\mu_{\log }(f)}}=\lim _{r_{n} \rightarrow+\infty} \frac{\log M\left(r_{n}, f\right)}{\left(\log r_{n}\right)^{\mu_{\log }(f)}}=\underline{\tau}_{\log }(f)
$$


and $\operatorname{lm}\left(E_{11}\right)=\int_{E_{11}} \frac{d r}{r}=\sum_{n=n_{1}}^{+\infty} \int_{\frac{n}{n+1} r_{n}}^{r_{n}} \frac{d t}{t}=\sum_{n=n_{1}}^{+\infty} \log \left(1+\frac{1}{n}\right)=+\infty$.

Lemma $2.14([\mathbf{9}])$. Let $\varphi:[0,+\infty) \rightarrow \mathbb{R}$ and $\psi:[0,+\infty) \rightarrow \mathbb{R}$ be monotone non-decreasing functions such that $\varphi(r) \leq \psi(r)$ for all $r \notin E_{12} \cup[0,1]$, where $E_{12} \subset(1,+\infty)$ is a set of finite logarithmic measure. Let $\gamma>1$ be a given constant. Then there exists an $r_{0}=r_{0}(\gamma)>0$ such that $\varphi(r) \leq \psi(\gamma r)$ for all $r>r_{0}$.

\section{Proofs of the Theorems.}

Proof of Theorem 1.1. Let $f \not \equiv 0$ be a meromorphic solution of (1.1). We suppose $\rho_{\log }(f)<\rho_{\log }\left(A_{s 0}\right)+1<+\infty$. We divide through equation (1.1) by $f\left(z+c_{s}\right)$ to get

$$
\begin{aligned}
\left|A_{s 0}(z)\right| \leq & \sum_{\substack{i=0 \\
i \neq s}}^{n} \sum_{j=0}^{m}\left|A_{i j}(z)\right|\left|\frac{f^{(j)}\left(z+c_{i}\right)}{f\left(z+c_{i}\right)}\right|\left|\frac{f\left(z+c_{i}\right)}{f\left(z+c_{s}\right)}\right| \\
& +\sum_{j=1}^{m}\left|A_{s j}(z)\right| \frac{f^{(j)}\left(z+c_{s}\right)}{f\left(z+c_{s}\right)} \mid .
\end{aligned}
$$

In relation to 1.2 and $(1.3)$, we set

$$
\rho=\max \left\{\rho_{\log }\left(A_{i j}\right):(i, j) \neq(s, 0)\right\},
$$

and

$$
\tau=\max \left\{\tau_{\log }\left(A_{i j}\right): \rho_{\log }\left(A_{i j}\right)=\rho_{\log }\left(A_{s 0}\right):(i, j) \neq(s, 0)\right\} .
$$

Then for a sufficiently large $r$, we have

$$
\left|A_{i j}(z)\right| \leq \exp \left\{(\log r)^{\rho+\varepsilon}\right\}, \quad(i, j) \neq(s, 0)
$$

if $\rho_{\log }\left(A_{i j}\right)<\rho_{\log }\left(A_{s 0}\right)$, and

$$
\left|A_{i j}(z)\right| \leq \exp \left\{(\tau+\varepsilon)(\log r)^{\rho_{\log }\left(A_{s 0}\right)}\right\}, \quad(i, j) \neq(s, 0)
$$

if $\rho_{\log }\left(A_{i j}\right)=\rho_{\log }\left(A_{s 0}\right)$. By Lemma 2.2 and Remark 2.2 , for any given $\varepsilon>0$ and $\alpha>1$, there exists a set $E_{2} \subset(1,+\infty)$ of finite logarithmic measure such that for all $|z|=r \notin[0,1] \cup E_{2}$, we have

$$
\begin{aligned}
\left|\frac{f^{(j)}\left(z+c_{i}\right)}{f\left(z+c_{i}\right)}\right| & \leq\left(\frac{(\log r)^{\rho_{\log }\left(f\left(z+c_{i}\right)\right)+\alpha+\varepsilon}}{r}\right)^{j} \\
& =\left(\frac{(\log r)^{\rho_{\log }(f)+\alpha+\varepsilon}}{r}\right)^{j} \quad(i=0,1, \cdots, n, j=1, \cdots, m) .
\end{aligned}
$$


By Lemma 2.8, there exists a set $E_{6} \subset(1,+\infty)$ of finite logarithmic measure such that for all $|z|=r \notin E_{6}$, we have for any given $\varepsilon>0$ and $\beta>1$

$$
\left|\frac{f\left(z+c_{i}\right)}{f\left(z+c_{s}\right)}\right| \leq \exp \left\{\frac{(\log r)^{\rho_{\log }(f)+\beta+\varepsilon}}{r}\right\}(i=0,1, \cdots, n, i \neq s) .
$$

Then we can choose an $\varepsilon>0$ sufficiently small to satisfy

$$
\tau+2 \varepsilon<\tau_{\log }\left(A_{s 0}\right), \quad \max \left\{\rho, \rho_{\log }(f)-1\right\}+2 \varepsilon<\rho_{\log }\left(A_{s 0}\right) .
$$

Substituting (3.2), (3.3), (3.4) and (3.5) into (3.1), for $|z|=r \notin[0,1] \cup E_{2} \cup E_{6}$, we get

$$
\begin{gathered}
M\left(r, A_{s 0}\right) \leq \exp \left\{\frac{(\log r)^{\rho_{\log }(f)+\beta+\varepsilon}}{r}\right\} O\left(\exp \left\{(\tau+\varepsilon)(\log r)^{\rho_{\log }\left(A_{s 0}\right)}\right\}\right. \\
\left.+\exp \left\{(\log r)^{\rho+\varepsilon}\right\}\right)\left(\frac{(\log r)^{\rho_{\log }(f)+\alpha+\varepsilon}}{r}\right)^{m},
\end{gathered}
$$

where $\left|A_{s 0}(z)\right|=M\left(r, A_{s 0}\right)$. By (3.6) and (3.7) and Lemma 2.14, we get

$$
\tau_{\log }\left(A_{s 0}\right)=\limsup _{r \rightarrow+\infty} \frac{\log M\left(r, A_{s 0}\right)}{(\log r)^{\rho_{\log }\left(A_{s 0}\right)}} \leq \tau+\varepsilon<\tau_{\log }\left(A_{s 0}\right)-\varepsilon
$$

which is a contradiction. Hence $\rho_{\log }(f) \geq \rho_{\log }\left(A_{s 0}\right)+1$.

Proof of the Theorem 1.2. Here, we use a method similar to the one in the proof of Theorem 1.1. Let $f \not \equiv 0$ be a meromorphic solution of (1.1). We suppose $\mu_{\log }(f)<\mu_{\log }\left(A_{s 0}\right)+1<+\infty$. In relation to 1.4 and 1.5$)$, we set

$$
\rho_{1}=\max \left\{\rho_{\log }\left(A_{s 0}\right):(i, j) \neq(s, 0)\right\},
$$

and

$$
\tau_{1}=\max \left\{\tau_{\log }\left(A_{i j}\right): \rho_{\log }\left(A_{s 0}\right)=\mu_{\log }\left(A_{s 0}\right):(i, j) \neq(s, 0)\right\} .
$$

Then for a sufficiently large $r$, we have

$$
\left|A_{i j}(z)\right| \leq \exp \left\{(\log r)^{\rho_{1}+\varepsilon}\right\}, \quad(i, j) \neq(s, 0)
$$

if $\rho_{\log }\left(A_{i j}\right)<\mu_{\log }\left(A_{s 0}\right)$, and

$$
\left|A_{i j}(z)\right| \leq \exp \left\{\left(\tau_{1}+\varepsilon\right)(\log r)^{\mu_{\log }\left(A_{s 0}\right)}\right\}, \quad(i, j) \neq(s, 0)
$$

if $\rho_{\log }\left(A_{i j}\right)=\mu_{\log }\left(A_{s 0}\right)$. By Remark 2.2. Lemma 2.9 and Lemma 2.10, for any given $\varepsilon>0, \alpha>1, \beta>1$, there exists a set $E_{8} \subset(1,+\infty)$ of infinite logarithmic measure such that for all $|z|=r \in E_{8}$, we have

$$
\left|\frac{f^{(j)}\left(z+c_{i}\right)}{f\left(z+c_{i}\right)}\right| \leq\left(\frac{(\log r)^{\mu_{\log }(f)+\alpha+\varepsilon}}{r}\right)^{j} \quad(i=0,1, \cdots, n, j=1, \cdots, m)
$$


and

$$
\left|\frac{f\left(z+c_{i}\right)}{f\left(z+c_{s}\right)}\right| \leq \exp \left\{\frac{(\log r)^{\mu_{\log }(f)+\beta+\varepsilon}}{r}\right\}(i=0,1, \cdots, n, i \neq s) .
$$

Then we can choose an $\varepsilon>0$ sufficiently small to satisfy

$$
\tau_{1}+2 \varepsilon<\underline{\tau}_{\log }\left(A_{s 0}\right), \quad \max \left\{\rho_{1}, \mu_{\log }(f)-1\right\}+2 \varepsilon<\mu_{\log }\left(A_{s 0}\right) .
$$

Substituting (3.8) 3.11) into 3.1), for $|z|=r \in E_{8}$, we get

$$
\begin{gathered}
M\left(r, A_{s 0}\right) \leq \exp \left\{\frac{(\log r)^{\mu_{\log }(f)+\beta+\varepsilon}}{r}\right\} O\left(\exp \left\{\left(\tau_{1}+\varepsilon\right)(\log r)^{\mu_{\log }\left(A_{s 0}\right)}\right\}\right. \\
\left.+\exp \left\{(\log r)^{\rho_{1}+\varepsilon}\right\}\right)\left(\frac{(\log r)^{\mu_{\log }(f)+\alpha+\varepsilon}}{r}\right)^{m},
\end{gathered}
$$

where $\left|A_{s 0}(z)\right|=M\left(r, A_{s 0}\right)$. By (3.12), (3.13) and Lemma 2.13, we get

$$
\underline{\tau}_{\log }\left(A_{s 0}\right)=\liminf _{\substack{r \rightarrow+\infty \\ r \in E_{8}}} \frac{\log M\left(r, A_{s 0}\right)}{(\log r)^{\mu_{\log }\left(A_{s 0}\right)}} \leq \tau_{1}+\varepsilon<\underline{\tau}_{\log }\left(A_{s 0}\right)-\varepsilon
$$

which is a contradiction. Hence $\mu_{\log }(f) \geq \mu_{\log }\left(A_{s 0}\right)+1$.

Proof of the Theorem 1.3. By Remark 1.3 , we know that $\rho_{\log }\left(A_{s 0}\right)=$ $\rho$. Let $f \not \equiv 0$ be a meromorphic solution of (1.1). We suppose $\rho_{\log }(f)<$ $\rho_{\log }\left(A_{s 0}\right)+1=\rho+1<+\infty$. By the assumptions of Theorem 1.3 , there is a set $H$ of complex numbers satisfying $\overline{\log d e n s}\{|z|: z \in H\}>0$ such that for $z \in H$, we have (1.6) and (1.7) as $|z|=r \rightarrow+\infty$. Set $H_{1}=\{r=|z|: z \in H\}$, since $\overline{\log d e n s}\{|z|: z \in H\}>0$. Then by Remark 2.1. for $H_{1}$ there is $\int_{H_{1}} \frac{d r}{r}=\infty$. Clearly, (3.4) and (3.5) hold for all $z$ satisfying $|z|=r \notin[0,1] \cup E_{2} \cup E_{6}$, where $E_{2}$ and $E_{6}$ are defined similarly as in the proof of Theorem 1.1. Substituting (1.6), 1.7), (3.4) and (3.5) into (3.1), for $|z|=r \in H_{1} \backslash[0,1] \cup E_{2} \cup E_{6}$, and any given $\varepsilon\left(0<\varepsilon<\frac{\rho-\rho_{\log }(f)+1}{2}\right)$, we get

$$
\begin{aligned}
\exp \left\{\alpha[\log r]^{\rho-\varepsilon}\right\} \leq & n \exp \left\{\beta[\log r]^{\rho-\varepsilon}\right\} \\
& \cdot \exp \left\{\frac{(\log r)^{\rho_{\log }(f)+\beta+\varepsilon}}{r}\right\}\left(\frac{(\log r)^{\rho_{\log }(f)+\alpha+\varepsilon}}{r}\right)^{m} .
\end{aligned}
$$

It follows that

$$
\exp \left\{(\alpha-\beta)[\log r]^{\rho-\varepsilon}\right\} \leq n \exp \left\{\frac{(\log r)^{\rho_{\log }(f)+\beta+\varepsilon}}{r}\right\}\left(\frac{(\log r)^{\rho_{\log }(f)+\alpha+\varepsilon}}{r}\right)^{m}
$$


By $0<\varepsilon<\frac{\rho-\rho_{\log }(f)+1}{2}$ and 3.14 , we obtain a contradiction. Hence we get $\rho_{\log }(f) \geq \rho+1=\rho_{\log }\left(A_{s 0}\right)+1$.

Proof of the Theorem 1.4. Let $f \not \equiv 0$ be a meromorphic solution of (1.1). If $\rho_{\log }(f)=\infty$, then the result is trivial. Now we suppose $\rho_{\log }(f)<+\infty$. We divide through equation (1.1) by $f\left(z+c_{s}\right)$ to get

$$
-A_{s 0}(z)=\sum_{\substack{i=0 \\ i \neq s}}^{n} \sum_{j=0}^{m} A_{i j}(z) \frac{f^{(j)}\left(z+c_{i}\right)}{f\left(z+c_{i}\right)} \frac{f\left(z+c_{i}\right)}{f\left(z+c_{s}\right)}+\sum_{j=1}^{m} A_{s j}(z) \frac{f^{(j)}\left(z+c_{s}\right)}{f\left(z+c_{s}\right)} .
$$

It follows

$$
\begin{aligned}
m\left(r, A_{s 0}\right) & \leq \sum_{\substack{i=0 \\
i \neq s}}^{n} \sum_{j=0}^{m} m\left(r, A_{i j}\right)+\sum_{j=1}^{m} m\left(r, A_{s j}\right) \\
& +\sum_{i=0}^{n} \sum_{j=1}^{m} m\left(r, \frac{f^{(j)}\left(z+c_{i}\right)}{f\left(z+c_{i}\right)}\right)+\sum_{\substack{i=0 \\
i \neq s}}^{n} m\left(r, \frac{f\left(z+c_{i}\right)}{f\left(z+c_{s}\right)}\right)+O(1) .
\end{aligned}
$$

Suppose that

$$
\frac{\sum_{(i, j) \neq(s, 0)} m\left(r, A_{i j}\right)}{m\left(r, A_{s 0}\right)}<1=\mu<\lambda<1 .
$$

Then for a sufficiently large $r$, we have

$$
\sum_{(i, j) \neq(s, 0)} m\left(r, A_{i j}\right)<\lambda m\left(r, A_{s 0}\right) .
$$

By Lemma 2.3, for a sufficiently large $r$ and any given $\varepsilon>0$, we have

$$
m\left(r, \frac{f\left(z+c_{i}\right)}{f\left(z+c_{s}\right)}\right)=O\left((\log r)^{\rho_{\log }(f)-1+\varepsilon}\right), i=0, \cdots, n, i \neq s .
$$

The logarithmic derivative lemma and Remark 2.2 lead to

$$
m\left(r, \frac{f^{(j)}\left(z+c_{i}\right)}{f\left(z+c_{i}\right)}\right)=O\left((\log (\log r))^{\rho_{\log }(f)-1+\varepsilon}\right), j=1, \cdots, m .
$$

Thus, by substituting (3.18), 3.19) and (3.20) into (3.16), for a sufficiently large $r$ and any given $\varepsilon>0$, we obtain

$$
m\left(r, A_{s 0}\right) \leq \lambda m\left(r, A_{s 0}\right)+O\left((\log r)^{\rho_{\log }(f)-1+\varepsilon}\right)+O\left((\log (\log r))^{\rho_{\log }(f)-1+\varepsilon}\right) .
$$


From (3.21), it follows that

$$
(1-\lambda) m\left(r, A_{s 0}\right) \leq O\left((\log r)^{\rho_{\log }(f)-1+\varepsilon}\right)+O\left((\log (\log r))^{\rho_{\log }(f)-1+\varepsilon}\right) .
$$

By (3.22), we obtain $\rho_{\log }(f) \geq \rho_{\log }\left(A_{s 0}\right)+1$. Thus, Theorem 1.4 is proved.

Proof of THE TheOREM 1.5, Let $f \not \equiv 0$ be a meromorphic solution of (1.1). If $\rho_{\log }(f)=\infty$, then the result is trivial. Now we suppose $\rho_{\log }(f)<+\infty$. Set

$$
\delta\left(\infty, A_{s 0}\right)=\liminf _{r \rightarrow+\infty} \frac{m\left(r, A_{s 0}\right)}{T\left(r, A_{s 0}\right)}=\delta>0 .
$$

Thus from (3.23), for a sufficiently large $r$, we have

$$
m\left(r, A_{s 0}\right)>\frac{1}{2} \delta T\left(r, A_{s 0}\right) .
$$

Thus, by substituting (3.19), (3.20) and (3.24) into (3.16), for a sufficiently large $r$ and any given $\varepsilon>0$, we obtain

$$
\begin{aligned}
\frac{\delta}{2} T\left(r, A_{s 0}\right)< & m\left(r, A_{s 0}\right) \leq \sum_{\substack{i=0 \\
i \neq s}}^{n} \sum_{j=0}^{m} m\left(r, A_{i j}\right)+\sum_{j=1}^{m} m\left(r, A_{s j}\right) \\
& +\sum_{i=0}^{n} \sum_{j=1}^{m} m\left(r, \frac{f^{(j)}\left(z+c_{i}\right)}{f\left(z+c_{i}\right)}\right)+\sum_{\substack{i=0 \\
i \neq s}}^{n} m\left(r, \frac{f\left(z+c_{i}\right)}{f\left(z+c_{s}\right)}\right)+O(1) \\
\leq & \sum_{\substack{i=0 \\
i \neq s}}^{n} \sum_{j=0}^{m} T\left(r, A_{i j}\right)+\sum_{j=1}^{m} T\left(r, A_{s j}\right)+O\left((\log r)^{\rho_{\log }(f)-1+\varepsilon}\right) \\
& +O\left((\log (\log r))^{\rho_{\log }(f)-1+\varepsilon}\right) .
\end{aligned}
$$

Since $\max \left\{\rho_{\log }\left(A_{i j}\right):(i, j) \neq(s, 0)\right\}<\rho_{\log }\left(A_{s 0}\right)$, then by Lemma 2.12, there exists a set $E_{10} \subset(1,+\infty)$ of infinite logarithmic measure such that

$$
\max \left\{\frac{T\left(r, A_{i j}\right)}{T\left(r, A_{s 0}\right)}: \quad(i, j) \neq(s, 0)\right\} \rightarrow 0, r \rightarrow+\infty, r \in E_{10} .
$$

Thus, by (3.25) and (3.26), for all $r \in E_{10}, r \rightarrow+\infty$, we have

$$
\left(\frac{\delta}{2}-o(1)\right) T\left(r, A_{s 0}\right) \leq O\left((\log r)^{\rho_{\log }(f)-1+\varepsilon}\right)+O\left((\log (\log r))^{\rho_{\log }(f)-1+\varepsilon}\right) .
$$

It now follows from (3.27) and Lemma 2.11 that $\rho_{\log }(f) \geq \rho_{\log }\left(A_{s 0}\right)+1$. Thus, Theorem 1.5 is proved. 
Proof of the Theorem 1.6, Let $f \not \equiv 0$ be a meromorphic solution of (1.1). If $\rho_{\log }(f)=\infty$, then the result is trivial. Now we suppose $\rho_{\log }(f)<+\infty$. As in the proof of Theorem 1.4, by substituting (3.18), (3.19) and (3.20) into (3.16), for a sufficiently large $r$ and any given $\varepsilon>0$, we have

$$
(1-\lambda) m\left(r, A_{s 0}\right) \leq O\left((\log r)^{\rho_{\log }(f)-1+\varepsilon}\right)+O\left((\log (\log r))^{\rho_{\log }(f)-1+\varepsilon}\right) .
$$

By Lemma 2.11, we have

$$
\lim _{\substack{r \rightarrow+\infty \\ r \in E_{9}}} \frac{\log T\left(r, A_{s 0}\right)}{\log \log r}=\rho_{\log }\left(A_{s 0}\right),
$$

where $E_{9}$ is a set of $r$ of infinite logarithmic linear measure. Since $\delta\left(\infty, A_{s 0}\right)=$ $\liminf _{r \rightarrow+\infty} \frac{m\left(r, A_{s 0}\right)}{T\left(r, A_{s 0}\right)}>0$, we obtain

$$
\lim _{\substack{r \rightarrow+\infty \\ r \in E_{9}}} \frac{\log m\left(r, A_{s 0}\right)}{\log \log r}=\rho_{\log }\left(A_{s 0}\right) .
$$

Thus, by (3.28) and (3.30), we obtain $\rho_{\log }(f) \geq \rho_{\log }\left(A_{s 0}\right)+1$. Thus, Theorem 1.6 is proved.

Acknowledgements. The author is grateful to the referee for his/her valuable comments which helped to improve this paper.

\section{References}

1. Belaïdi B., Growth of meromorphic solutions of finite logarithmic order of linear difference equations, Fasc. Math., 54 (2015), 5-20.

2. Belaïdi B., Some properties of meromorphic solutions of logarithmic order to higher order linear difference equations, submitted.

3. Cao T. B., Liu K., Wang J., On the growth of solutions of complex differential equations with entire coefficients of finite logarithmic order, Math. Reports, 15(65), 3 (2013), 249269.

4. Chen Z. X., Shon K. H., On growth of meromorphic solutions for linear difference equations, Abstr. Appl. Anal., 2013, Art. ID 619296, 1-6.

5. Chern P. T. Y., On meromorphic functions with finite logarithmic order, Trans. Amer. Math. Soc., 358 (2006), no. 2, 473-489.

6. Chiang Y. M., Feng S. J., On the Nevanlinna characteristic of $f(z+\eta)$ and difference equations in the complex plane, Ramanujan J., 16 (2008), no. 1, 105-129.

7. Goldberg A., Ostrovskii I., Value Distribution of Meromorphic functions, Transl. Math. Monogr., 236, Amer. Math. Soc., Providence RI, 2008.

8. Gundersen G. G., Estimates for the logarithmic derivative of a meromorphic function, plus similar estimates, J. London Math. Soc. (2), 37 (1988), no. 1, 88-104.

9. Gundersen G. G., Finite order solutions of second order linear differential equations, Trans. Amer. Math. Soc., 305 (1988), no. 1, 415-429. 
10. Halburd R. G., Korhonen R. J., Difference analogue of the lemma on the logarithmic derivative with applications to difference equations, J. Math. Anal. Appl., 314 (2006), no. 2, 477-487.

11. Halburd R. G., Korhonen R. J., Nevanlinna theory for the difference operator, Ann. Acad. Sci. Fenn. Math., 31 (2006), no. 2, 463-478.

12. Hayman W. K., Meromorphic functions, Oxford Mathematical Monographs Clarendon Press, Oxford 1964.

13. Heittokangas J., Wen Z. T., Functions of finite logarithmic order in the unit disc, Part I. J. Math. Anal. Appl., 415 (2014), no. 1, 435-461.

14. Heittokangas J., Wen Z. T., Functions of finite logarithmic order in the unit disc, Part II. Comput. Methods Funct. Theory, 15 (2015), no. 1, 37-58.

15. Laine I., Yang C. C., Clunie theorems for difference and q-difference polynomials, J. Lond. Math. Soc. (2), 76 (2007), no. 3, 556-566.

16. Tu J., Yi C. F., On the growth of solutions of a class of higher order linear differential equations with coefficients having the same order, J. Math. Anal. Appl., 340 (2008), no. 1, 487-497.

17. Wen Z. T., Finite logarithmic order solutions of linear q-difference equations, Bull. Korean Math. Soc., 51 (2014), no. 1, 83-98.

18. Wu S. Z., Zheng X. M., Growth of meromorphic solutions of complex linear differentialdifference equations with coefficients having the same order, J. Math. Res. Appl., 34 (2014), no. 6, 683-695.

19. Yang C. C., Yi H. X., Uniqueness theory of meromorphic functions, Mathematics and its Applications, 557, Kluwer Academic Publishers Group, Dordrecht, 2003.

20. Zheng X. M., Tu J., Growth of meromorphic solutions of linear difference equations, J. Math. Anal. Appl., 384 (2011), no. 2, 349-356.

Received March 29, 2016

Department of Mathematics

Laboratory of Pure and Applied Mathematics

University of Mostaganem (UMAB)

B. P. 227 Mostaganem

Algeria

e-mail: benharrat.belaidi@univ-mosta.dz 